\title{
A Modified Dummy Plaque for the Accurate Placement of Ruthenium-106 Plaques in Brachytherapy of Intraocular Tumours
}

\author{
Jørgen Krohn
}

Section of Ophthalmology, Department of Clinical Medicine, University of Bergen, and Department of Ophthalmology, Haukeland University Hospital, Bergen, Norway

\section{Key Words}

Uveal melanoma - Episcleral brachytherapy .

Ruthenium-106 plaque · Transillumination · Indirect ophthalmoscopy

\begin{abstract}
Purpose: To present a new technique to ensure the correct positioning of ruthenium plaques in episcleral brachytherapy. Materials and Methods: An acrylic dummy plaque is made opaque by sanding both sides with sandpaper, and its edge is covered by a black marking tape. This modified plaque is temporarily sutured to the sclera overlying the choroidal tumour site. The tip of an endoillumination probe is placed at the anterior edge of the plaque, yielding a strong light scattering within the opaque acrylic material. Due to the light-absorbing tape around the plaque border, the scattered light is confined within the plaque, and its perimeter can be observed by indirect ophthalmoscopy as a circle of transilluminated light surrounding the tumour. When the correct position has been found, the dummy plaque is replaced by a ruthenium-106 plaque. Results: The technique was successfully applied in 5 patients with posterior choroidal melanoma. Compared to standard focal transillumina-
\end{abstract}

tion, its main advantage is that the position of the entire plaque and tumour can be observed simultaneously in one field without any movement or manipulation of the light probe or plaque. Conclusion: The described transillumination technique and modified dummy plaque facilitate the correct positioning of ruthenium plaques in brachytherapy of choroidal melanoma.

(c) 2015 S. Karger AG, Basel

\section{Introduction}

Correct dosimetry and accurate placement of the radioactive plaques are the two most important factors to achieve local tumour control and reduce the risk of radiation-induced side effects in brachytherapy of uveal melanoma. Different techniques have been employed to ensure correct plaque placement during and after surgery, such as ultrasound [1-4], magnetic resonance imaging $[5,6]$, and plaque-mounted diode lights $[7,8]$. Damato et al. [9] have developed a dummy plaque with small holes for the insertion of a transilluminator while performing intraoperative indirect ophthalmoscopy. The most commonly used technique, however, is to

\section{KARGER}

E-Mail karger@karger.com www.karger.com/oop
(C) 2015 S. Karger AG, Basel

2296-4681/15/0021-0001\$39.50/0
Jørgen Krohn

Department of Ophthalmology

Haukeland University Hospital

NO-5021 Bergen (Norway)

E-Mail jorgen.krohn@ @else-bergen.no 
check the actual position of the plaque by indirect ophthalmoscopy and transillumination while moving or indenting a fibre optic light probe along the margins of the plaque $[10,11]$.

Our routine approach for correct placement of the radioactive plaques has been transscleral transillumination of light reflected from the inner surface of dummy plaques (without silicone inserts) observed by indirect ophthalmoscopy [12]. This requires bowl-shaped plaques with sidewalls, like the standard Collaborative Ocular Melanoma Study (COMS) plaques. Ruthenium plaques, due to their smooth and concave inner surface, are not suitable for this procedure. In our department, both iodine and ruthenium are routinely used in brachytherapy of uveal melanoma: iodine for the larger and ruthenium for the smaller tumours. The work presented here describes a novel technique to ensure the correct positioning of ruthenium-106 plaques in episcleral brachytherapy of uveal melanoma, based on simple modifications of a conventional acrylic dummy plaque and the use of transscleral transillumination in conjunction with indirect ophthalmoscopy.

\section{Materials and Methods}

A transparent acrylic dummy plaque with a diameter of 15 $\mathrm{mm}$ (ACD.A24; BEBIG GmbH, Berlin, Germany) is made opaque by sanding both sides of the plaque with 180 -grit sandpaper, and its edge is covered by a $2.5-\mathrm{mm}$-wide, black marking tape (MKT015; Clinipak Ltd., High Wycombe, UK) (fig. 1). This tape is normally used for marking surgical instruments and is therefore resistant to various detergents and sterilisation procedures. The modified dummy plaque is temporarily sutured to the sclera overlying the choroidal tumour site. The tip of a 20 -gauge fibre optic endoillumination probe is then placed exactly at the anterior edge of the plaque (between the two suture eyelets), yielding a strong light scattering within the opaque acrylic material. Due to the presence of the black, light-absorbing tape around the plaque border, the scattered light is confined within the plaque (fig. 2). We prefer to use a light source that can emit yellowish light with a wavelength of $515 \mathrm{~nm}$ (Xenon Brightstar; DORC, Zuidland, The Netherlands), as it penetrates the tissue more easily and deeply than light of shorter wavelengths [13]. Indirect ophthalmoscopy is then performed through a Quadraspheric contact lens (Volk Optical Inc., Mentor, Ohio, USA) and a surgical microscope equipped with a slit lamp. By turning off the microscope light and increasing the fibre optic light to a maximum, the perimeter of the plaque can easily be observed as a circle of transilluminated light surrounding the tumour. The technique enables the surgeon to determine the exact position of the plaque and to make the necessary adjustments in relation to the tumour, macula, and optic disc. When the correct position has been found, the dummy plaque is replaced by a ruthenium-106 plaque.
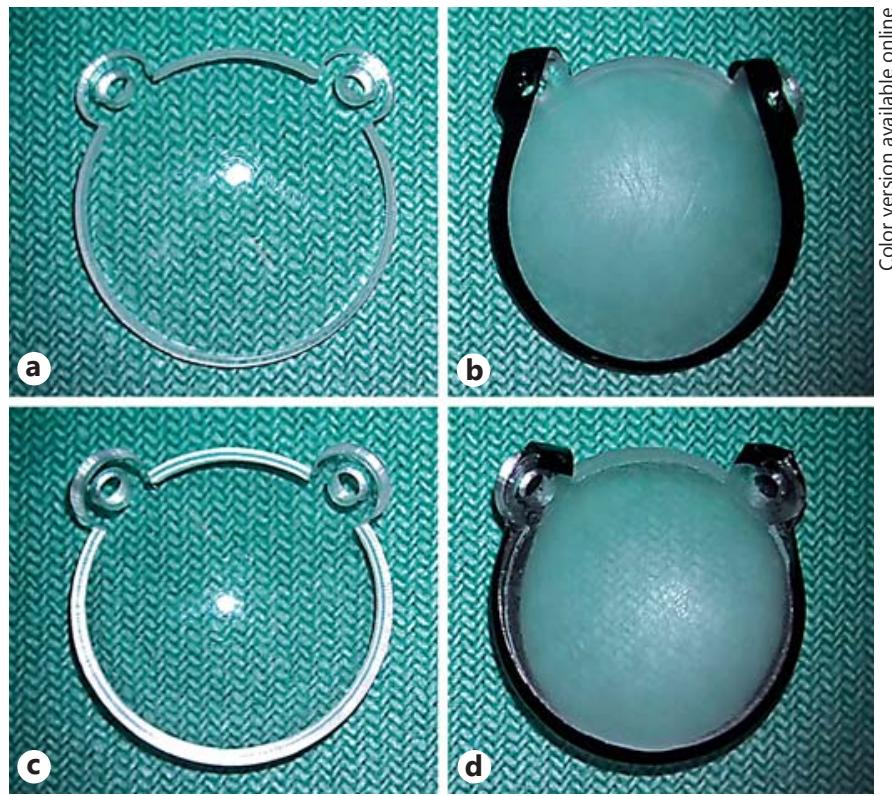

Fig. 1. The outer surface of a transparent acrylic dummy plaque (a), and a modified, opaque acrylic dummy plaque where the margin is covered by black marking tape (b). The inner surface of the same transparent acrylic dummy plaque (c), and the modified, opaque acrylic dummy plaque (d). Note that the tape covers the edge of the plaque.

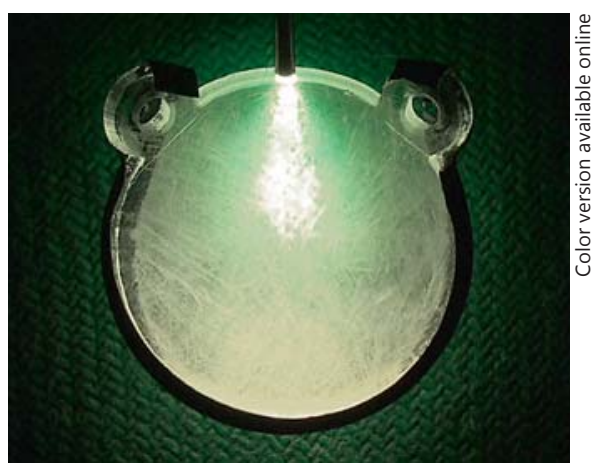

Fig. 2. The inner surface of a modified, opaque acrylic dummy plaque. A 20 -gauge fibre optic light probe is placed against the edge of the plaque. Note that the light is strongly scattered and confined within the opaque acrylic material.

Between October 2013 and June 2014, this technique has been used in 5 patients with choroidal melanoma located posterior to the equator. The study was registered and approved by the Regional Committee for Medical and Health Research Ethics, Western Norway, and followed the official ethical regulations for clinical research and the Declaration of Helsinki. All patients gave their written informed consent prior to participation. 

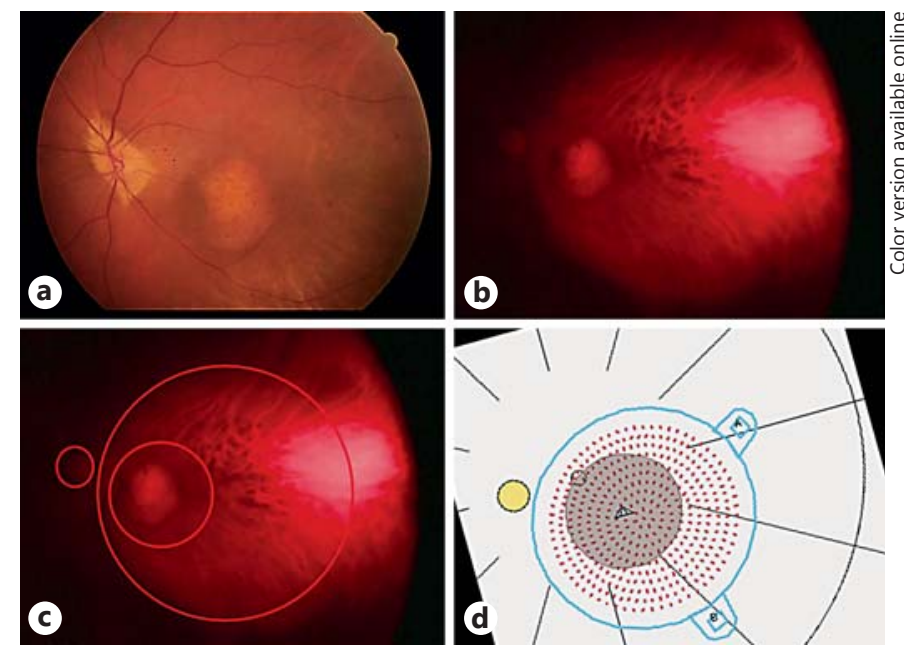

Fig. 3. a Fundus photograph of a small, centrally located, amelanotic choroidal melanoma in the left eye of a 69-year-old woman. b Intraoperative image showing transillumination of light from the dummy plaque. Note that the contour of a suture eyelet can be distinguished in the upper right part of the image. $\mathbf{c}$ The circles illustrate the location of the optic disc, the tumour, and the perimeter of the dummy plaque. $\mathbf{d}$ The location of the dummy plaque corresponds closely with that in the retinal diagram of the Plaque Simulator (BEBIG GmbH).

\section{Results}

The case series included 4 women and 1 man, with a mean age at the time of surgery of 66 years (range 4177). Three melanomas were pigmented and two were amelanotic. The mean largest basal tumour diameter was $10.0 \mathrm{~mm}$ (range 7.5-12.0), and the mean apical tumour height was $4.3 \mathrm{~mm}$ (range 3.0-6.5). The modification of the dummy plaque and the surgical procedure were easy to perform, and the tape remained strongly attached to the plaque during surgery.

In all 5 patients, plaque placement was greatly facilitated by the use of the modified, opaque dummy plaque and transscleral transillumination. Due to the posterior tumour localisation, close to the macula and optic nerve, accurate plaque positioning was particularly important. The ophthalmoscopic view through the surgical microscope was of good diagnostic quality, making it possible to determine and adjust the position of the plaque in relation to the rim of the tumour, the fovea, and the optic disc. Posterior tilting of the plaque resulted in a more diffuse demarcation of the transilluminated light. A fundus photograph and fundus images captured from an intraoperative video recorded during indirect ophthalmoscopy are shown and compared with the treatment plan and the retinal diagram of the Plaque Simulator (BEBIG GmbH) in figure 3. None of the patients experienced negative side effects.

\section{Discussion}

The present paper describes how to modify and use a conventional acrylic dummy plaque to ensure the correct positioning of ruthenium plaques during brachytherapy of posterior choroidal melanoma. The main advantage of this technique, compared to the use of focal transillumination along the margin of a silver dummy plaque, is that the position of the entire plaque and tumour can be observed simultaneously in one field without any movement or manipulation of the light probe or plaque. This is particularly advantageous for tumours located in the posterior pole of the eye, because it reduces the risk of damage to the many ciliary vessels and nerves that pierce the sclera in this region. Although no direct comparisons were made, it is our impression that it is easier to achieve a correct plaque position by the use of the modified, transparent dummy plaque compared to a standard silver dummy plaque.

The technique may also be used for more anteriorly located tumours, but is then limited by the restricted view of the peripheral fundus during indirect ophthalmoscopy. For tumours located anterior to the equator, ordinary transocular or transpupillary transillumination, casting a tumour shadow on the sclera, is more suitable for accurate tumour localisation and plaque placement [14].

In summary, the modified dummy plaque enhances transillumination and thus its visualisation during indirect ophthalmoscopy. This makes it possible to determine and even photographically document the location of the dummy plaque in relation to the tumour and vital structures of the posterior segment, thereby facilitating the correct positioning of ruthenium plaques in episcleral brachytherapy.

\section{Statement of Ethics}

The study was registered and approved by the Regional Committee for Medical and Health Research Ethics, Western Norway. All patients gave their written informed consent prior to participation. 


\section{References}

-1 Pavlin CJ, Japp B, Simpson ER, McGowan HD, Fitzpatrick PJ: Ultrasound determination of the relationship of radioactive plaques to the base of choroidal melanomas. Ophthalmology 1989;96:538-542.

$>2$ Harbour JW, Murray TG, Byrne SF, Hughes JR, Gendron EK, Ehlies FJ, Markoe AM: Intraoperative echographic localization of iodine 125 episcleral radioactive plaques for posterior uveal melanoma. Retina 1996;16: 129-134.

$>3$ Finger PT, Romero JM, Rosen RB, Iezzi R, Emery R, Berson A: Three-dimensional ultrasonography of choroidal melanoma: localization of radioactive eye plaques. Arch Ophthalmol 1998;116:305-312.

-4 Tabandeh H, Chaudhry NA, Murray TG, Ehlies F, Hughes R, Scott IU, Markoe AM: Intraoperative echographic localization of iodine-125 episcleral plaque for brachytherapy of choroidal melanoma. Am J Ophthalmol 2000;129:199-204.
5 Houdek PV, Schwade JG, Medina AJ, Poole CA, Olsen KR, Nicholson DH, Byrne S, Quencer R, Hinks RS, Pisciotta V: MR technique for localization and verification procedures in episcleral brachytherapy. Int J Radiat Oncol Biol Phys 1989;17:1111-1114.

6 Hanna SL, Lemmi MA, Langston JW, Fontanesi J, Brooks HL Jr, Gronemeyer S: Treatment of choroidal melanoma: MR imaging in the assessment of radioactive plaque position. Radiology 1990;176:851-853.

$>7$ Finger PT, Iezzi R, Romero JM, Rosen RB, Szechter A, Hegde H: Plaque-mounted diode-light transillumination for localization around intraocular tumors. Arch Ophthalmol 1999;117:179-183.

8 Finger PT, Iezzi R, Esteveo ML, Szechter A, Rosen RB, Berson A: Diode-light transillumination for ophthalmic plaque localization around juxtapapillary choroidal melanomas. Int J Radiat Oncol Biol Phys 1999;44:887-890.

$\checkmark 9$ Damato B, Patel I, Campbell IR, Mayles HM, Errington RD: Local tumor control after ${ }^{106} \mathrm{Ru}$ brachytherapy of choroidal melanoma. Int J Radiat Oncol Biol Phys 2005;63:385-391.
0 Robertson DM, Fuller DG, Anderson RE: A technique for accurate placement of episcleral iodine-125 plaques. Am J Ophthalmol 1987; 103:63-65.

11 Snyder WB, Fuller DG, Fish GE: An inexpensive fiberoptic light pipe to aid in placement of episcleral radioactive plaques. Ophthalmic Surg 1988;19:62-63.

12 Krohn J, Seland JH, Monge OR, Rekstad BL: Transillumination for accurate placement of radioactive plaques in brachytherapy of choroidal melanoma. Am J Ophthalmol 2001; 132:418-419.

13 Krohn J, Xu CT, Svenmarker P, Khoptyar D, Andersson-Engels S: Transscleral visible/nearinfrared spectroscopy for quantitative assessment of melanin in a uveal melanoma phantom of ex vivo porcine eyes. Exp Eye Res 2010; 90:330-336.

14 Trichopoulos N, Damato B: Examination techniques; in Singh AD, Damato B, Pe'er J, Murphree AL, Perry JD (eds): Clinical Ophthalmic Oncology. Philadelphia, SaundersElsevier, 2007, pp 171-174. 